\title{
Silver Staining of Lily Microsporocytes during Meiotic Prophase
}

\author{
Toshio Ohyama and Michio Ito \\ Department of Biology, Faculty of Science, Nagoya University, Nagoya 464, \\ Japan
}

\begin{abstract}
Nuclei of lily microsporocytes in meiotic prophase were subjected to two-dimensional spreading and stained with silver nitrate. This method made it possible to observe the behavior of synaptonemal complexes with light microscopy. Axial cores of homologous chromosomes, which had been formed at the leptotene stage, were paired during the zygotene stage, consequently being integrated into the synaptonemal complexes. During the middle zygotene stage, a number of paired segments were observed as heavily stained stretches in the spread nuclei, thus indicating the multiple initiation of pairings along sets of homologous chromosomes.
\end{abstract}

Synapsis and crossing-over between homologous chromosomes take place during the meiotic prophase in eukaryotic organisms. It has been considered that synapsis plays significant roles in recombinational events, because the accurate pairing of homologous chromosomes is prerequisite to the crossing-over. The pairing of homologous chromosomes is maintained by synaptonemal complexes (SCs), which are tripartite structures composed of two lateral elements and a central element (21).

Thin-sectioned or microspread preparations of SCs have been extensively studied with the electron microscope, and information about the fine structure and the behavior of SCs during meiotic prophase have been accumulated for various organisms (reviewed in 10, 20,21,32). Recently, it has become possible to observe wholemounted SCs under a light microscope by the use of a silver-staining method. Although this method was initially improved for the benefit of the visualization of nucleolar organizer regions in mammalian chromosomes $(3,11)$, it has been advantageously applied to the cytogenetic analysis of the synapsis of homologous chromosomes in mammalian spermatocytes $(5,6,8,9,22,23)$.

The application of this method to plant meiocytes is, however, limited, because most kinds of microsporocytes are enclosed in thick callose walls which make it difficult to spread the nuclei on glass slides for silver staining. To overcome this problem, it is necessary to digest the walls with enzymes. Microsporocytes in solanaceous plants (27), Allium, Secale (1), Rhoeo (28), and some plants including lily (2) have been available for silver-staining method.

Microsporocytes of liliaceous plants can be cultured in vitro through the normal meiotic process (29), and it is easy to prepare their protoplasts (16). In particular, lily microsporocytes are preferable materials for cytological and biochemical studies on SCs, not only do the meiotic processes proceed with a high degree of synchrony, but they contain a relative abundance of SCs in their nuclei. 
This report describes a simple and reproducible method of silver staining for lily microsporocytes. Using this method, we can avoid the difficulties in precisely determining the meiotic stages, such as the transition from leptonema to zygonema. In addition, it is expected that silver staining will be a useful and convenient procedure for the isolation of SCs, because alternate procedures utilizing biochemical activities and antibodies specific to SCs have not yet been made available. Although the details of SC formation have already been reported by Moens (19) and Holm (12) in electron microscopic studies, the behavior of whole-mounted SCs in lily microsporocytes has not yet been described. It is necessary to survey the change of appearance of silverpositive structures for the benefit of further biochemical studies on SCs. Therefore, we report the sequential analysis of the pairing process visualized by silver staining under a light microscope.

\section{MATERIALS AND METHODS}

Microsporocytes were obtained from two hybrid lilies, Enchantment and Black Beauty, grown in a greenhouse. The approximate stages of the microsporocytes were estimated from the correlation between bud length and meiotic development (7). Extrusion of microsporocytes were carried out according to the procedure described previously (29). Some of the extruded cells were fixed with a mixture of ethanol : acetic acid $(3: 1)$ and stained with propionic orcein for the precise identification of the meiotic stages.

The isolation of protoplasts was performed by the procedure of Ito (16), with a slight modification. Meiotic cells extruded from a bud were suspended in White's medium containing $0.35 \mathrm{M}$ sucrose, and washed several times with the same medium. The enzyme solution for the digestion of cell walls contained $4 \%$ (w/v) Cellulase Onozuka $565 \mathrm{~N}$ and $2 \%$ (w/v) Macerozyme R-10 (Kinki Yakult CO., LTD, JAPAN) in White's medium, pH 5.7. The meiocytes were suspended in the enzyme solution, and were incubated at $25^{\circ} \mathrm{C}$ for $1 \mathrm{~h}$ with gentle agitation. Protoplasts were collected by a low-speed centrifugation and washed twice with White's medium containing $0.4 \mathrm{M}$ sucrose. The protoplasts were gently suspended in $100 \mu \mathrm{l}$ of $0.001 \%$ Triton X-100 solution, and were incubated at room temperature for $5 \mathrm{~min}$. After the incubation period, $100 \mu \mathrm{l}$ of $8 \%$ formalin solution buffered with $10 \mathrm{mM}$ borate at $\mathrm{pH} 8.1$ were added to the suspension, and the mixture was chilled in an ice bath for $10 \mathrm{~min}$. Then, $40 \mu \mathrm{l}$ of the mixture were placed on a slide, spread with the aid of a plastic pipette tip, and allowed to air-dry at room temperature. The slide was rinsed in $5 \mathrm{mM}$ borate buffer ( $\mathrm{pH}$ 9) for $30 \mathrm{~min}$ to remove the fixative, and briefly air-dried at room temperature. The staining method employed in this study was a silver nitrate incubation technique improved by Bloom and Goodpasture (3). The specimen was flooded with $50 \mu 1$ of $50 \%$ $(w / w)$ silver nitrate and covered with a coverslip. The silver nitrate was dissolved in distilled water just before use. The specimen was then placed in a moist plastic chamber, and incubated at $55^{\circ} \mathrm{C}$ for $15 \mathrm{~h}$ in the dark. After the incubation period, the slide was rinsed with distilled water and air-dried. Stained samples were mounted in euparal and observed under a light microscope.

Fig. 1. The nuclei during meiotic prophase visualized by silver staining in microsporocytes of Lilium, hybrid var. Enchantment. A. early leptotene. Arrows indicate the segments of axial cores; B. late leptotene; C. early zygotene. Arrowheads indicate representatives of paired part of axial cores.; D. middle zygotene; E. late zygotene; F. pachytene; G. early diplotene; H. diplotene. Bar represents $10 \mu \mathrm{m}$. 

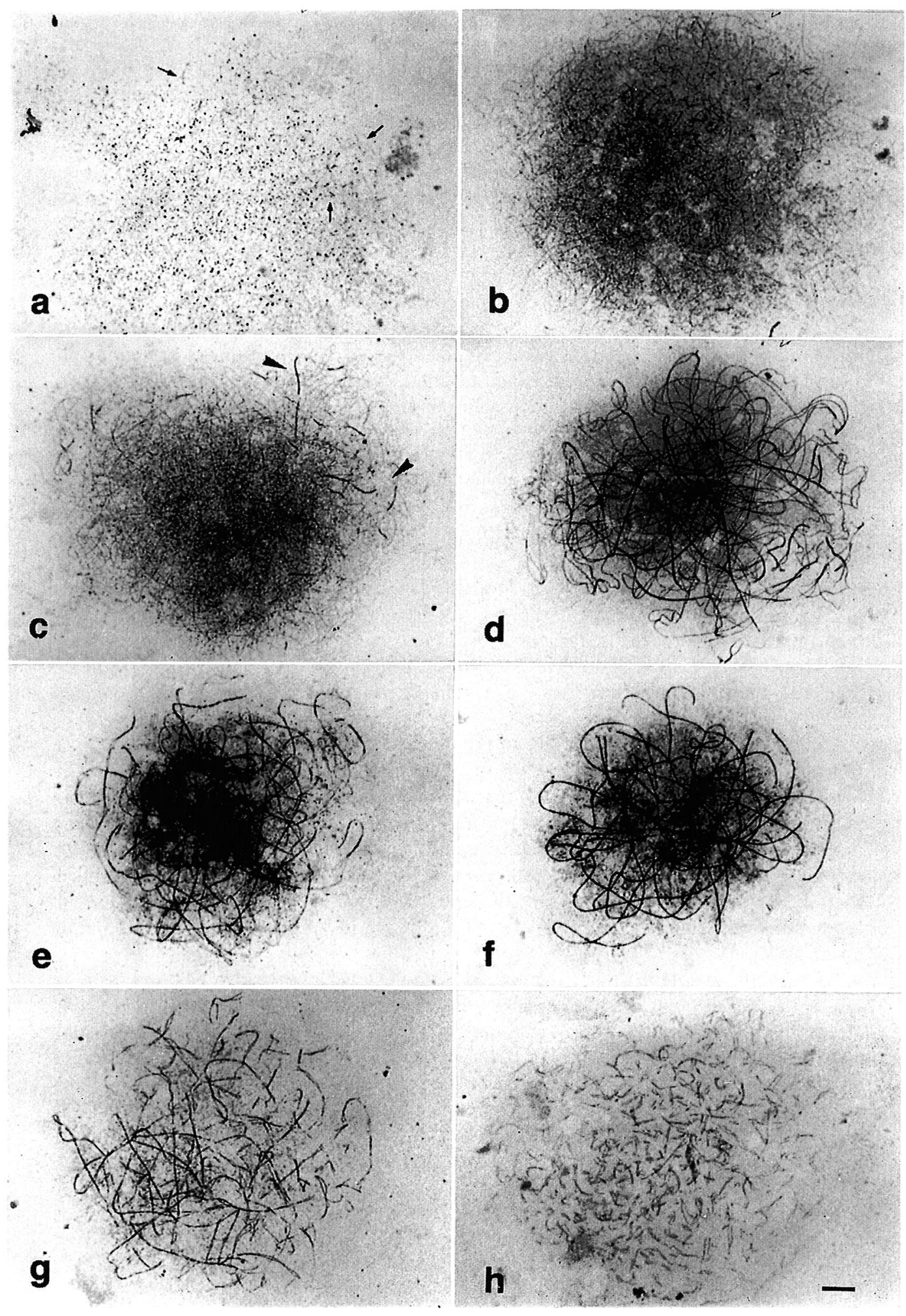


\section{RESULTS AND DISCUSSION}

When microsporocytes at the premeiotic G2 phase were subjected to silver staining, no distinctive structures were observed in the spread nuclei. In the nucleus which had entered into the leptotene stage, fibrous structures were recognized (Fig. 1a), seeming to correspond to the axial cores described by Moens (19). In the early stage of the leptotene, axial cores appeared to be segmental rather than of a thread-like structure. From the late leptotene stage to the early zygotene stage, the axial cores were distinctively observed as continuous structures along the chromosomes (Fig. 1b). In the zygotene stage, the pairing between each axial core of homologues had commenced, and paired parts were detected as densely stained segments in spread nuclei (Fig. 1c). Using an orcein-staining method or other methods employed for staining chromosomes, it was difficult to distinguish the paired parts of homologous chromosomes. Silver staining visualized paired parts of axial cores as densely stained segments, and allowed us to determine the precise meiotic stage. In the progress of meiotic stages to the middle zygotene stage, the increment in the number of paired segments was obvious, and the total number of paired segments was ranging from 50 to 70 in a nucleus, regardless of its length (Fig. 1d). As the number of chromosomes in lily is 12 in haploid, these observations indicated that synapsis initiates at multiple points in a pair of homologous chromosomes. Moens (19) has already reported that SC formation is initiate at several places along a set of homologues in lily microsporocytes. Although we could not count more than 70 pieces of paired segments in one nucleus under a light microscope, Holm (12) showed the presence of 170 pieces of SC segments in a nucleus at the zygotene stage in L. longiflorum by a three-dimensional reconstruction analysis of electron micrographs. Similar observations were reported in Secale microsporocytes (1), and also in mouse spermatocytes (5). Therefore, the multiple initiation may be a general aspect of SC formation in higher organisms.

Unfortunately, lily chromosomes in the meiotic prophase were so long that it was difficult to distinguish each chromosome, even in a well-spread preparation. No localization of initiation sites or direction of pairing on each set of homologous chromosomes was recognizable. The pairing, however, seemed to be initiate asynchronously at each initiation site, because paired segments detected by silver staining increased in number during the zygotene stage (Fig. 1c, d). In addition, it was doubtful that the pairing was initiated from the ends of homologous chromosomes. Paired segments were frequently observed at proximal positions of axial cores rather than the distal positions.

At the late zygotene stage, the paired segments became elongated, and, simultaneously, the number of short segments decreased (Fig. 1e). Pairing was completed along the full length of the chromosomes by the pachytene stage, and unpaired axial cores were no longer observed (Fig. 1f). Lily microsporocytes proceed in the meiotic development with a high degree of synchrony in a bud up to the pachytene stage, but the synchrony is disturbed after the pachytene stage. At the early diplonema, the disjunction of paired chromosomes proceeds rapidly, in contrast with the synaptic process. Therefore, it was difficult to precisely determine the behavior of axial cores through the late prophase. In the nucleus at early diplonema, unpaired parts of axial cores were recognizable by silver staining (Fig. 1g). As chromosome condensation proceeded, axial cores gradually disappeared, reducing the stainability by silver nitrate (Fig. 1h). When cells entered into diakinesis, no further distinctive structures 


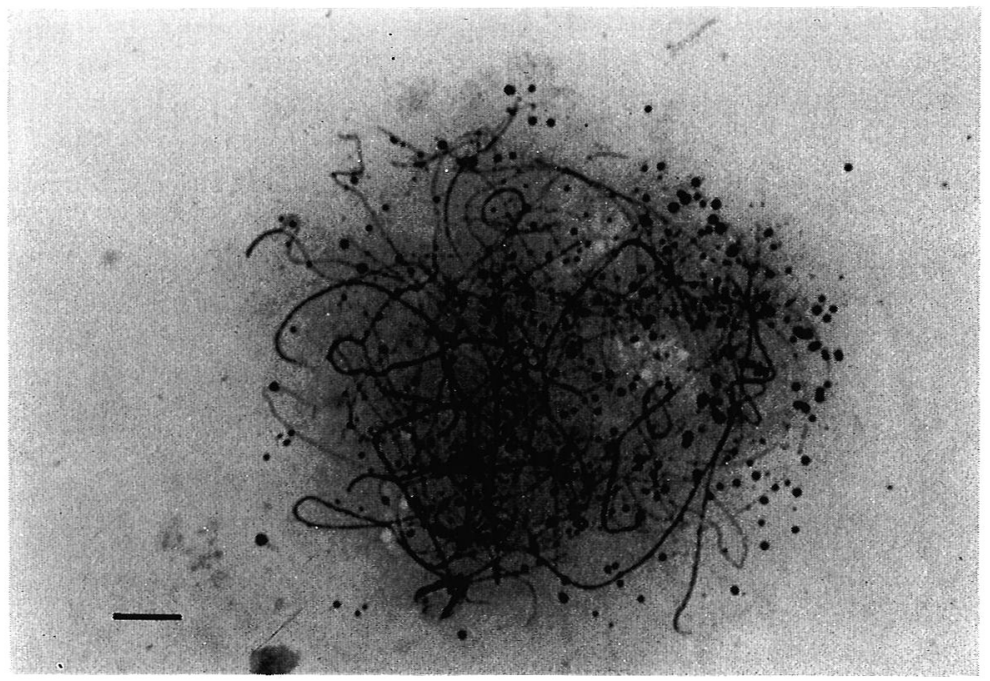

Fig. 2. Silver stained pachytene microsporocyte from the achiasmatic hybrid lily, Black Beauty. Bar represents $10 \mu \mathrm{m}$.

were observed. Lily microsporocytes cultured in vitro normally progressed through the meiotic prophase, the process of SC formation being the same as that of in vivo, as described above.

The microsporocytes of Black Beauty, a highly achiasmatic hybrid lily, show an incomplete pairing that is attributed to poor homology between each set of homologous chromosomes (30). The pairing pattern at the pachytene stage in this lily was similar to that of the zygonema of chiasmatic lilies, such as Enchantment (Fig. 2). In Black Beauty, however, paired segments were markedly fewer than those in the middle zygonema of Enchantment, and short segments were not observed in the nucleus. This observation suggested that the pairing at homologous sites had been already accomplished by this stage.

In lily microsporocytes, small amounts of DNA are synthesized during the zygotene stage; this DNA is known as zygotene DNA (13). Zygotene DNA synthesis is considered to play significant roles in meiosis, especially in the pairing of homologous chromosomes and in meiotic development $(15,25)$. Electron microscope autoradiography has revealed that zygotene DNA synthesis take place close to, or within SCs (17). When microsporocytes were cultured in the presence of an inhibitor of DNA synthesis, SC formation was effectively interrupted (25). For instance, the cells in leptonema explanted into White's medium containing $2 \mathrm{mM}$ deoxyadenosine showed the arrest of meiotic development at the early zygotene stage. When such arrested cells were subjected to silver staining, axial cores were completely formed along the chromosomes, and the profile of pairing was the same as that of the early zygotene stage. The pairing of the axial cores did not progress during prolonged cultures in the presence of deoxyadenosine (data not shown). Therefore, it is considered that the zygotene DNA synthesis acts on the pairing process of axial cores, but not on the formation of axial cores itself.

Synaptonemal complexes are known to play significant roles in genetic recombination. Despite the morphological and histochemical studies performed on SCs thus 
far, sufficient information concerning their biochemical nature has not yet been obtained, so as to determine the fundamental functions of SCs at the molecular level. From cytochemical analysis, it is noted that the SCs are constituted mainly of protein, and small amount of nucleic acids $(4,26)$. Recently, the isolation of SCs was attempted in mammal spermatocytes $(14,18,31)$ and in moth oocytes $(24)$, with the aid of a procedure developed for the preparation of nuclear matrices or chromosome scaffold. However, distinctive molecular components which are prominent in SC construction have not yet been defined. The use of lily microsporocytes has the advantage in that it is easy to collect pachynema of an adequate purity. The examination of the isolation of residual nuclear structures, including SCs, form lily microsporocytes for biochemical studies has been carried out with the aid of the silver-staining method (submitted).

Acknowledgements. This work was supported by a Grant-in-Aid from the Ministry of Education, Science and Culture of Japan (Nos. 59340043 and 61540501).

\section{REFERENCES}

1. Albini, S.M., G.H. Jones and B.M.N. Wallace. A method for preparing two-dimensional surface-spreading of synaptonemal complexes from plant meiocytes for light and electron microscopy. Exp. Cell Res. 152, 280-285, 1984

2. Anderson, L.K., S.M. Stack, M.H. Fox and Z. Chaunshan. The relationship between genome size and synaptonemal complex length in higher plants. Exp. Cell Res. 156, 367-378, 1985

3. Bloom, S.E. and C. Goodpasture. An improved technique for selective silver staining of nucleolar organizer regions in human chromosomes. Hum. Genet. 34, 199-206, 1976

4. Comings, D.E. and T.A. OKadA. Fine structure of the synaptonemal complex: Regular and stereo electron microscopy of deoxyribonuclease treated whole-mount preparations. Exp. Cell Res. 65, 104-116, 1971

5. Dietrich, A.J.J. and R.J.P. Mulder. A light microscopic study of the development and behavior of the synaptonemal complex in spermatocytes of the mouse. Chromosoma 83, 409418,1980

6. Dresser, M.E. and M.J. Moses. Silver staining of synaptonemal complexes in surface spreads for light and electron microscopy. Exp. Cell Res. 121, 416-419, 1979

7. ERICKSON, R.O. Cytological and growth correlation in the flower buds and another of Lilium longiflorum. Amer. J. Bot. 35, 729-739, 1948

8. Fletcher, J.M. Light microscope analysis of meiotic prophase chromosomes by silver staining. Chromosoma 72, 241-248, 1979

9. ForeJt, J. and P. GoETz. Synaptonemal complexes of mouse and human pachytene chromosomes visualized by silver staining in air-dried preparations. Chromosoma 73, 255-261, 1979

10. Gillies, C.B. Synaptonemal complex and chromosome structure. Ann. Rev. Genet. 9, 91-109, 1978

11. Goodpasture, C. and S.E. Bloom. Visualization of nucleolar organizer regions in mammalian chromosomes using silver staining. Chromosoma 53, 37-50, 1975

12. Holm, P.B. Three-dimensional reconstruction of chromosome pairing during the zygotene stage of meiosis in Lilium longiflorum (Thunb). Carlsberg Res. Commun. 42, 103-151, 1977

13. Hotta, Y. and H. Stern. Analysis of DNA synthesis during meiotic prophase in Lilium. J. Mol. Biol. 55, 337-355, 1971

14. Ierard, L.A., S.B. Moss and A.R. BellvÉ. Synaptonemal complexes are integral components of the isolated mouse spermatocyte nuclear matrix. J. Cell Biol. 96, 1717-1726, 1983

15. Ito, M., Y. Hotta and H. Stern. Studies of meiosis in vitro II. Effect of inhibiting DNA synthesis during meiotic prophase on chromosome structure and behavior. Develop. Biol. 16, 54-77, 1967 
16. Iто, M. Studies on the behavior of meiotic protoplasts I. Isolation from microsporocytes of liliaceous plants. Bot. Mag. Tokyo 86, 133-141, 1973

17. Kurata, N. and M. Ito. Electron microscope autoradiography of ${ }^{3} \mathrm{H}$-thymidine incorporation during the zygotene stage in microsporocytes of lily. Cell Struct. Funct. 3, 349-356, 1978

18. Li, S., M.L. Meistrich, W.A. Brock, T.C. Hsu and M.T. Kuo. Isolation and preliminary characterization of the synaptonemal complex from rat pachytene spermatocytes. Exp. Cell Res. 144, 63-72, 1983

19. Moens, P.B. The structure and function of the synaptonemal complex in Lilium longiflorum sporocytes. Chromosoma 23, 418-451, 1968

20. Moens, P.B. Ultrastructural studies of chiasma distribution. Ann. Rev. Genet. 12, 433-450, 1978

21. Moses, M.J. Synaptonemal complex. Ann. Rev. Genet. 2, 363-412, 1968

22. Pathak, S. and T.C. Hsu. Silver-stained structures in mammalian meiotic prophase. Chromosoma 70, 195-203, 1979

23. Pathak, S., Y.F. LaU and H.L. Drwinga. Observations on the synaptonemal complex in armenian hamster spermatocytes by light microscopy. Chromosoma 73, 53-60, 1979

24. Raveh, D. and A. Ben-Ze'Ev. The synaptonemal complex as part of the nuclear matrix of the flour moth, Ephestia kuehniella. Exp. Cell Res. 153, 99-108, 1984

25. Rотн, T.F. and M. Ito. DNA-dependent formation of the synaptonemal complex at meiotic prophase. J. Cell Biol. 35, 247-255, 1967

26. Solari, A.J. Ultrastructure and composition of the synaptonemal complex in spread and negatively stained spermatocytes of the golden hamster and the albino rat. Chromosoma 39 , 237-263, 1972

27. Stack, S. Two-dimensional spreads of synaptonemal complexes from solanaceous plants. I. The technique. Stain Technology 57, 265-272, 1982

28. Stack, S.M. and D.L. Soulliere. The relation between synapsis and chiasma formation in Rhoeo spathacea. Chromosoma 90, 72-83, 1984

29. Takegami, M.H., M. Yoshioka, I. TanaKa and M. Ito. Characteristics of isolated microsporocytes from liliaceous plants for studies of the meiotic cell cycle in vitro. Plant Cell Physiol. 22, 1-10, 1981

30. Toledo, L.A., M.D. Bennet and H. Stern. Cytological investigations of the effect of colchicine on meiosis in Lilium hybrid cv. "Black Beauty' microsporocytes. Chromosoma 72, 157-173, 1979

31. WALMSLEY, M. and M.J. Moses. Isolation of synaptonemal complexes from hamster spermatocytes. Exp. Cell Res. 133, 405-411, 1981

32. Wettstein, D., S.W. Rasmussen and P.B. Holm. Synaptonemal complex in genetic segregation. Ann. Rev. Genet. 18, 331-413, 1984

(Received for publication, March 5, 1987) 